\title{
Open Versus Arthroscopic Treatment of Chronic Lateral Epicondylitis and Worker's Compensation
}

\author{
Laxminarayan Bhandari, M.D., Fadi Bouri, M.D., and Tuna Ozyurekoglu, M.D.
}

Purpose: To compare the short-term outcomes between arthroscopic and open procedures for the treatment of lateral epicondylitis. Because a significant portion of patients have worker's compensation (WC), the outcomes in these subgroups were separately analyzed. Methods: A retrospective analysis of patients who had surgical treatment of lateral epicondylitis by a single surgeon from 2010 to 2017 was performed. Patients who were symptomatic after 9 months of conservative treatments including nonsteroidal medications, steroid injections, and physical therapy were offered surgical intervention. The type of surgery was selected after detailed consultation with the patient. Charts were reviewed for preoperative pain, grip strength as measured by dynamometer, smoking status, and WC status. Postoperative assessments included pain and grip strength at 6 weeks and time taken to return to full duty. Two groups were compared using unpaired $t$ test and chi-squared tests. Results were compared with similar studies in the literature. Results: The study included 30 patients in the arthroscopic debridement group and 42 patients in the open tenotomy and reinsertion group. Eighteen patients $(25 \%)$ had WC. Compared with the open group, the arthroscopic group had earlier return to full duty (mean 7.13 weeks, confidence interval [CI] 6.21 to 8.05 versu mean of 12.22 weeks, CI 11.21 to $13.24 ; P<.001$ ) and less time for complete pain relief (mean 7.4 weeks, CI 7.02 to 7.93 versus 9.5 , CI 8.68 to $10.44 ; P=.043$ ). No difference was seen among the groups in terms of unfavorable outcome (persistent pain and recurrence of pain), JAMAR hand dynamometer score at 6 weeks, and visual analog score at 6 weeks. A total of 11 patients (15\%) had unfavorable outcome. Incidence of unfavorable outcomes was more in patients with WC insurance $36 \%$ in WC versus $7 \%$ in non-WC; $P=.023)$. No association was seen with smoking status. WC patients also had a longer time to return to full duty ( 16.68 weeks for WC versus 7.65 weeks for non-WC; $P<.001$ ) and a longer time to get complete pain relief $(12.4$ weeks for WC versus 7.5 weeks for non-WC; $P<.001)$. Conclusion: The arthroscopic technique offers advantages of earlier return to work and shorter recovery period along with additional advantages of joint inspection and ability to treat coexisting pathologies. WC patients had a longer time to return to full duty and time for complete pain relief. Level of Evidence: Level III, retrospective comparative study.

$\mathbf{L}$ ateral epicondylitis (LE) is an overuse disease involving the extensor muscles of the forearm. It can be a debilitating condition, especially for the working population. Commonly referred to as "tennis elbow," this

From the Christine M Kleinert Institute of Hand and Microsurgery, Louisville, KY, U.S.A.

The authors report that they have no conflicts of interest in the authorship and publication of this article. Full ICMJE author disclosure forms are available for this article online, as supplementary material.

Received January 16, 2020; accepted July 15, 2020.

Address correspondence to Laxminarayan Bhandari, M.D., Chritine M Kleinert Institute of Hand and Microsurgery, 225 Abraham Flexner Way, Ste 700,Louisville, KY 40202,U.S.A.E-mail:lax321@gmail.comorlbhandari@ cmki.org

(C) 2020 THE AUTHORS. Published by Elsevier Inc. on behalf of the Arthroscopy Association of North America. This is an open access article under the CC BY-NC-ND license (http://creativecommons.org/licenses/by-nc-nd/4.0/). 2666-061X/2058

https://doi.org/10.1016/j.asmr.2020.07.010 disease can advance to degenerative tears. ${ }^{1}$ The relative hypervascularity of the lateral epicondyle area, vascular dysfunction, eccentric contractions, and unrecognized shoulder pathology are factors that contribute to LE. ${ }^{2,3}$

Many treatment modalities have been described including rest, nonsteroidal anti-inflammatory drugs, physical therapy, and braces. Injections with steroids, alcohol, carbolic acid, botulinum toxin, and plateletrich plasma have also been tried with varying success. $^{2}$ Most cases of LE are self-limiting and respond well to nonoperative management. However, $\sim 4 \%$ to $11 \%$ of patients have persistent pain with failed conservative treatment and require surgical intervention. ${ }^{4}$ Surgical interventions may include release of extensor carpi radialis brevis (ECRB) attachment, ${ }^{2}$ debridement of pathologic tissue, ${ }^{1}$ denervation of lateral epicondyle, ${ }^{5}$ lengthening of extensor origin, ${ }^{6}$ excision of lateral epicondyle, and anconeus rotation. ${ }^{7}$ Although various authors have reported successful results with different 
techniques, there is no consensus on the best surgical technique to manage LE. $^{8}$

The surgical approach can be open, percutaneous, or arthroscopic. ${ }^{2}$ Although the arthroscopic technique allows for inspecting the elbow joint and diagnosing and treating concomitant pathologies, using minimal incisions, the open technique is quicker and cheaper. ${ }^{9,10}$ Several studies have compared arthroscopic and open approaches for surgical management of LE. $^{9-17}$ Most ${ }^{9,1-16}$ have focused on long-term outcomes and reported equal outcomes for open and arthroscopic approaches. There is little data on short-term outcomes. Because LE is usually a self-limiting condition, short-term outcomes are important. Although 3 studies ${ }^{11,12,16}$ have evaluated time to return to work, none have investigated time taken for complete pain relief.

The aim of our study was to compare arthroscopic and open procedures in terms of time to return to full duty and time taken for complete pain relief to assess the short-term outcomes. We also evaluated the risk factors for unfavorable outcome and complications. Our hypothesis was that there would be no difference in shortterm outcomes between the open and arthroscopic groups, similar to the observations of long-term studies. A review of the literature for similar studies was done.

\section{Materials and Methods}

Institutional review board approval was obtained for this retrospective comparative study. The study reviewed patients over a 7-year period from 2010 to 2017. Patients who underwent surgery for lateral epicondylitis by the senior author (O.T.) and had $\geq 3$ months of follow up were included. Patients undergoing simultaneous surgeries for multiple conditions and patients lost to follow-up were excluded.

Patients with the clinical diagnosis of LE who were still symptomatic despite 9 months of conservative treatment were offered surgery. These patients had received nonsteroidal drugs, steroid injection, and physical therapy. In addition, magnetic resonance imaging was performed in all patients. Both open and arthroscopic treatments were offered to the patients, and selection was made after discussion regarding the advantages and disadvantages of both procedures.

\section{Arthroscopic Technique}

The patients were operated in a lateral decubitus position under axillary block and general anesthesia. The superior anteromedial portal was developed as a viewing portal, and the superior anterolateral portal was used for synovectomy, limited capsulectomy, and removal of bone spurs. The direct anterolateral portal was used for common extensor tenotomy.

A diamond-shaped capsulectomy was performed without violating the anterior border of the ECRB tendon or the line connecting the radial head and the capitellum posteriorly. The ECRB origin was shaved close to the lateral epicondyle. The healthy shiny tendon fibers were preserved, whereas the dull-colored diseased tendon fibers were removed (Fig 1).

The posterior part of the joint was also evaluated using the superior posterolateral portal. The posterolateral capsule and any synovial fringe or synovial fold present were removed through the soft spot portal to examine the radial head in pronation and supination to complete the procedure. The arm was placed in a bulky Jones dressing, and a wrist brace was worn for 4 to 6 weeks.

\section{Open Technique}

The patients were operated in a supine position under axillary block. A $5-\mathrm{cm}$ incision was made over the lateral epicondyle. The superficial fibers of the extensor digitorum communis muscle were divided to expose the origin of the ECRB tendon. The degenerated or partially torn ECRB tendon origin was debrided of any calcification and dull fibrin deposits. Bone spurs on the ridge and the thickened and avascular cortex on the lateral epicondyle were removed with a rongeur (Fig 2). Three transosseous bone tunnels, in an anterolateral to posterior direction, were made across the lateral epicondyle using a 0.062 Kirschner wire. A no. 2 Fiberwire ${ }^{\circledR}$ was passed through the tunnels and ECRB tendon to reattach the tendon on the lateral epicondyle using 2 sutures tied posteriorly. The extensor digitorum communis and the fascia were also repaired upon closure. A long arm splint was used for postoperative care followed by a hinged elbow brace and a wristlet for 6 weeks.

\section{Data Collection}

Charts were reviewed for preoperative pain, score on a dynamometer (referred to as JAMAR), smoking status, and worker's compensation (WC) status. Postoperatively, pain and JAMAR at 6 weeks, as well as the time taken to return to full duty and time taken for complete pain relief, were noted in both groups. Both groups were compared using Student's $t$ test and chi-squared test to calculate the $P$ value.

Short-term outcomes were defined as the time taken to complete pain relief (TPR) and time taken for return to full duty (TFD). Unsatisfactory outcome was defined as either (a) persistence of pain for $>4$ months or (b) recurrence of pain within a year. Incidence of unsatisfactory outcome was calculated in both groups, and further subgroup analysis was done to find any association of the short-term outcomes with WC or smoking status.

\section{Statistical Methods}

A power analysis was done using previously published ${ }^{9-17}$ mean and standard deviation, obtaining a 
Fig 1. Arthroscopic procedure. The patient is in a lateral decubitus position with the upper arm over a paint roll support. The scope is on the superior anteromedial portal, the shaver on the direct anterolateral portal. (A) Capsulectomy is done (red arrows). (B) The tear (yellow arrow), scar, and deposits (yellow arrowheads) are observed. (C) The ECRB tendon origin (green arrows) is removed. (D) Posterolateral capsulectomy and synovectomy $\left({ }^{*}\right)$. The radial head $(\mathrm{R})$ is observed in pronation and in supination hand toward the upper right corner.

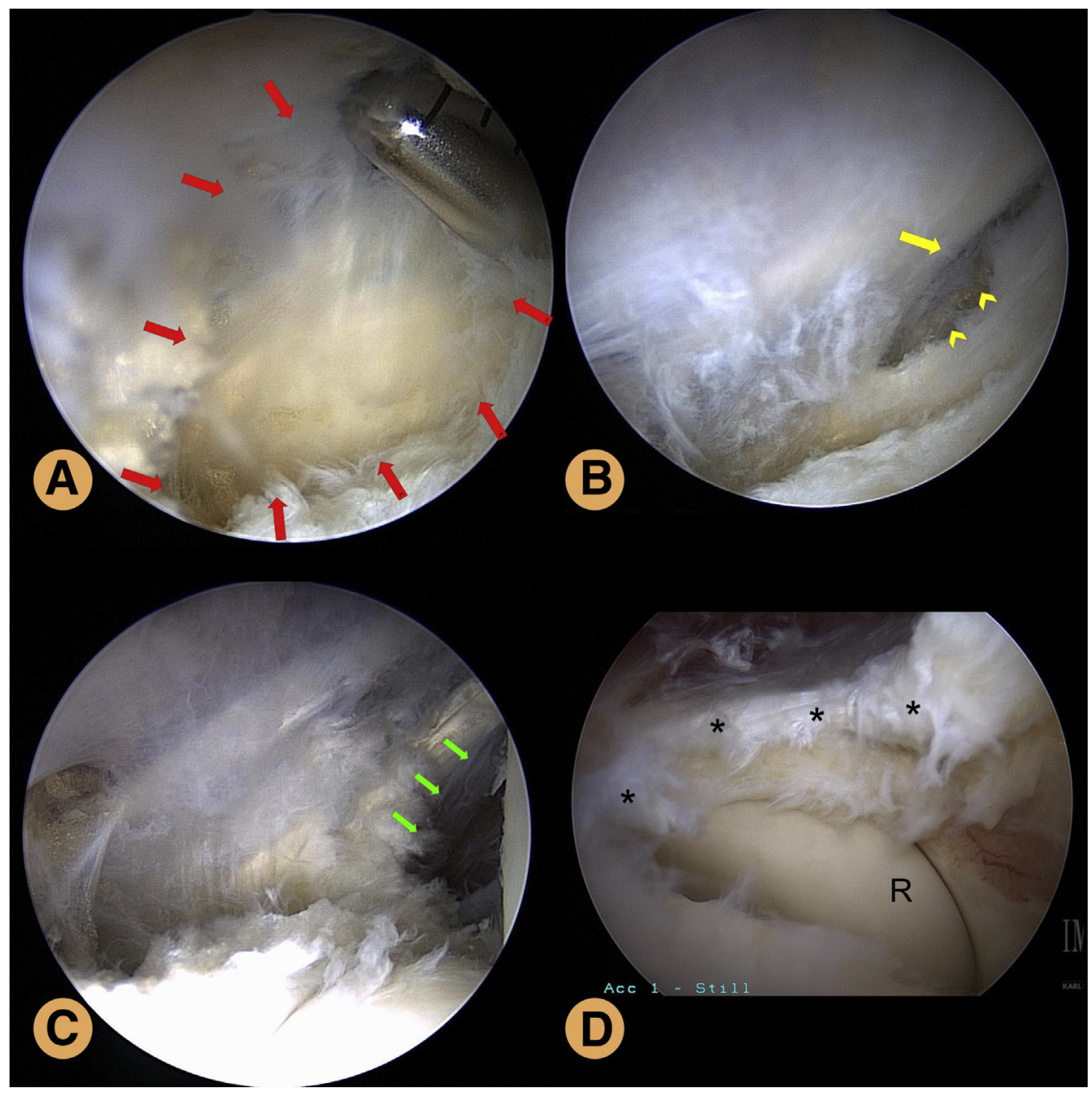

sample size of 60 . The means of open and arthroscopic groups were compared by $t$ test. The subgroups of WC, smokers, and unfavorable results were compared using chi-squared test. Multivariate testing (Pillai, Wilks, Hotelling-Lawley, Roy) was done on interaction terms in a linear model to see whether any interactions were significant.

\section{Results}

There were 30 patients in the arthroscopic group and 42 patients in the open surgery groups (age 28 to 67 years, 32 male and 40 female). There was no statistically significant difference between the groups in terms of age $(P=.07)$ sex $(P=.5)$, or preoperative visual analog score (VAS) $(P=.3)$. The open group had more smokers (26 of 42 in open versus 9 of 30 in arthroscopic; $P=.015$ ) and more WC patients (15 of 42 in open versus 3 of 30 in arthroscopic; $P=.02$ ).

The results were analyzed to see whether there was any difference in various outcome measures. TFD, an important outcome often asked by the patients preoperatively, was significantly less in the arthroscopic group (mean 7.13 weeks, confidence interval [CI] 6.21 to 8.05) compared with the open group (mean 12.22, CI 11.21 to $13.24, P<.001$ ). On further analysis, TFD for the WC group was significantly more than the nonWC group (16.68 versus 7.65 weeks, $P<.001$ ).

Smokers returned to full duty at 11.26 weeks, and nonsmokers at 9.17 weeks. This difference was not found to be significant $(P=.175)$. Although the TPR was less among nonsmokers, it did not reach statistical significance $(8.32$ versus 8.77 weeks, $P=.671)$.

Another frequently asked question by patients is the time taken for complete pain relief. TPR was found to be significantly less in the arthroscopic group (mean 7.4 weeks, CI 7.02 to 7.93 in arthroscopic versus mean 9.5, CI 8.68 to 10.44 in open, $P=.043$ ) and among non-WC patients $(7.5$ weeks in non-WC versus 12.4 weeks in WC, $P<.001$.$) .$

JAMAR assessment was done at 6 weeks in the affected extremity. Because all patients have different baseline JAMAR scores, the 6-week JAMAR value was compared with the preoperative value, and the difference was taken. Both positive and negative differences were noted. There was no significant difference in JAMAR change at 6 weeks between the 2 groups 


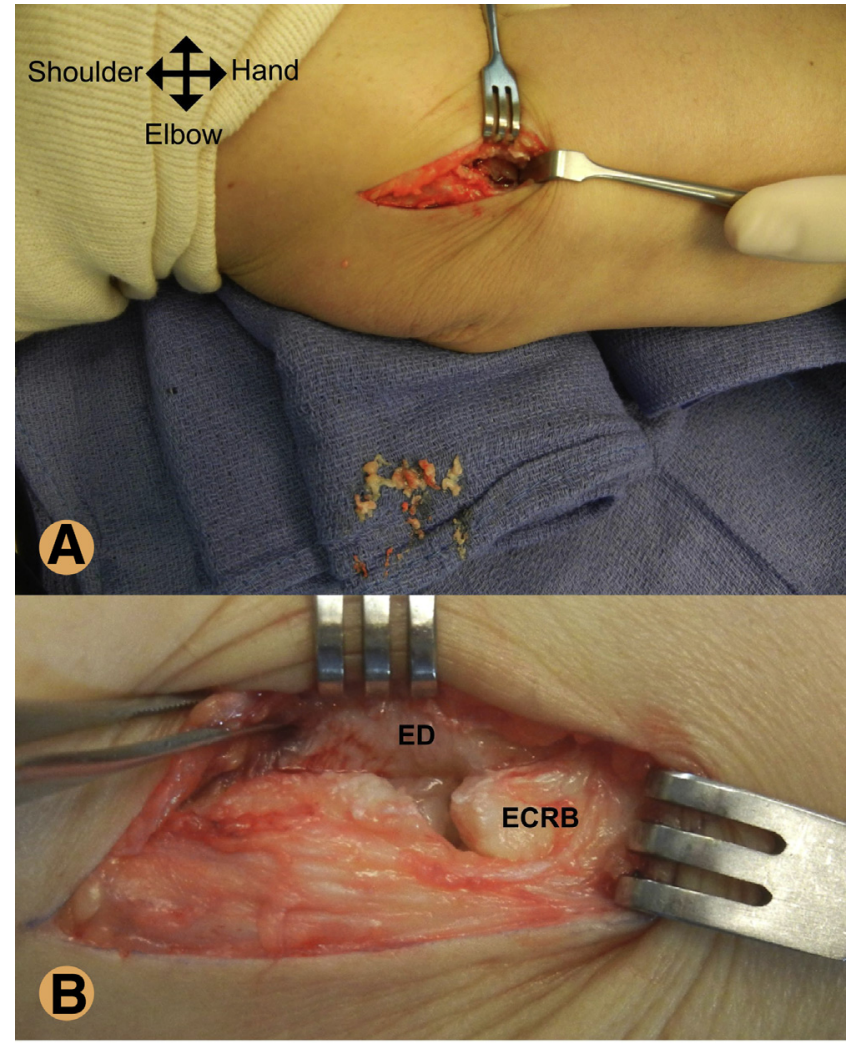

Fig 2. Open procedure. The patient is in supine position with elbow flexion and forearm pronation. (A) The surgical site and debrided degenerate tissue. (B) Closer look of extensor carpi radialis brevis (ECRB) origin following debridement. Extensor digitorum (ED) is retracted.

(arthroscopic group, mean 7.96 , CI 3.12 to 12.79 versus open group, mean $4.21 \mathrm{CI}-0.59$ to $9.02 ; P=.299$ ). Type of surgery, smoking status, and WC status did not produce any difference in JAMAR change at 6 weeks. Similar to JAMAR change at 6 weeks, there was no significant difference between the groups for VAS at 6 weeks (arthroscopic group, mean 3.15, CI 2.71 to 3.58 versus open group, mean 3.13, CI 2.63 to 3.62; $P=.488)$

Unfavorable outcomes were either persistence of pain or recurrence of pain. Persistent pain was defined as presence of continuous pain for 4 months after surgery. In the arthroscopic group, there were no patients with persistence of pain, whereas in the open group, there were 5 patients with persistent pain. Two of the 5 patients underwent a second surgery at a later date, one for flexor carpi radialis contracture, and the other for coronoid fracture at the proximal radioulnar joint. One patient never improved and ended up being assigned permanent l-handed duty. Another patient recovered slowly and went to full duty at 6 months. There was 1 patient who had thoracic outlet-related symptoms and was discharged on permanent light duty disability.

Recurrence of pain was defined as patient recurrence of pain within a year after a pain-free initial period. In the arthroscopic group, 4 patients who had recurrence of pain were managed with triamcinolone injections. They responded well to the steroid injection. In the open group, there were 2 patients with recurrence of pain, and 1 received triamcinolone injection and responded. When both persistent pain and recurrence of pain were taken together, there was no statistical significance between the type of surgery (4 of 30 in arthroscopic versus 7 of 42 in open; $P=.69$ ) or smoking status $(20 \%$ versus $10.8 \%, P=.449)$. However, a statistically significant difference was found between WC and non-WC groups $(38.8 \%$ versus $7.4 \% ; P=.0045)$. No significance found for smoking or surgery type. An effect modification was present with WC status: significant interactions were found between type of work and WC status $(P=.0126)$ and age and WC status $(P<.001)$. All results are tabulated in Table 1.

\section{Discussion}

We found that both surgical procedures were equally efficacious, with no significant difference between the incidence of unfavorable results, JAMAR change at 6 weeks, and VAS at 6 weeks. These 2 surgical procedures, done by a single surgeon, were compared on 3 variables: type of surgery, smoking status, and WC status. The short-term outcomes that were compared across the 3 variables were the time taken to full duty (TFD), time taken for complete pain relief (TPR), incidence of unfavorable results, JAMAR change at 6 weeks, and VAS at 6 weeks.

However, there were certain differences noted in the TFD and TPR. These short-term parameters are undoubtedly patient centric. Patients are most likely concerned about postoperative pain duration and when they can return to work.

Table 1. Comparison of groups

\begin{tabular}{|c|c|c|c|c|c|c|}
\hline \multirow[b]{2}{*}{ Measurement } & \multicolumn{2}{|c|}{ Arthroscopic Versus Open } & \multicolumn{2}{|c|}{ Smoking Versus Nonsmoking } & \multicolumn{2}{|c|}{ WC Versus Non-WC } \\
\hline & Value & $P$ Value & Value & $P$ Value & Value & $P$ Value \\
\hline TPR (wk) & 7.4 vs 9.5 & .043366. & 8.77 vs 8.32 & .671 & 12.4 vs 7.5 & $<.001$ \\
\hline TFD (wk) & 7.13 vs 12.22 & $<.001$ & 11.26 vs 9.17 & .175 . & 16.68 vs 7.65 & $<.001$ \\
\hline Unfavorable results (\%) & 13 vs 16 & .69 & 20 vs 10.8 & .449 & 38.8 vs 7.4 & .0045 \\
\hline JAMAR difference at $6 \mathrm{wk}$ & 7.9 vs 4.2 & .299 & 4.13 vs 8.7 & .302 & 1.9 vs 7.97 & .264 \\
\hline VAS at $6 \mathrm{wk}$ & 3.13 vs 3.15 & .488 & 3.11 vs 2.98 & .433 & 3.44 vs 3.03 & .286 \\
\hline
\end{tabular}


Table 2. Review of similar studies

\begin{tabular}{|c|c|c|c|c|c|}
\hline Reference & \multicolumn{3}{|c|}{ Group } & $P$ Value & Comments \\
\hline Stapleton and Baker $1996^{9}$ & & & & & $\begin{array}{l}\text { The authors reported that patients with } \\
\text { arthroscopic surgery returned to work and } \\
\text { sports faster; however, no objective data were } \\
\text { presented. Return to full activity was not } \\
\text { documented. Follow-up }>2 \text { y }\end{array}$ \\
\hline \multicolumn{6}{|l|}{ Parameters assessed } \\
\hline Failure & $1(20 \%)$ & $2(20 \%)$ & & & \\
\hline
\end{tabular}

Peart et al. $2004^{11}$

n

Parameters assessed

Good or excellent outcome

Return to work (mo)
33

$72 \%$

1.7
No significant differences between the 2 groups were noted. The authors reported that patients treated with arthroscopic release returned to work earlier.
Parameters assessed

Follow-up VAS for pain ( 1 to 6 )

Subjective functional outcome ( 1 to 6 )

Time taken off work (wk)

Roles and Maudsley score

Morrey score

Szabo et al. $2006^{1}$

n

Parameters assessed

Preoperative Andrew Carson score

Postoperative Andrew Carson score

Preoperative VAS at rest

Postoperative VAS at activity

Postoperative VAS at worst

Recurrence

Failure
54

$69 \%$

2.5

Both groups showed similar degrees of pain relief and no complications. Arthroscopic and open groups were operated by different surgeons; the assignment to endoscopic or open surgery depended on the surgeon available the day of surgery. Average follow-up $92.8 \mathrm{mo}$

$\begin{array}{ccc}20 & 10 & >.05 \\ 1.95 & 2.6 & >.05 \\ 1.85 & 2.5 & >.05 \\ 3.3 & 3 & >.05 \\ 1.7 & 2 & >.05 \\ 93.2 & 87.5 & \end{array}$

$\begin{array}{cc}41 & 38 \\ 158.9 & 160.2 \\ 195.4 & 195.3 \\ 5.2 & 5.4 \\ 9.5 & 9.5 \\ 1 & 1.2 \\ 2 & 5 \\ 1 & 2\end{array}$

No significant differences noted between the groups in terms of outcome. Follow-up 24 to 108 mo

\begin{tabular}{|c|c|c|}
\hline \multirow{2}{*}{\multicolumn{3}{|c|}{$\begin{array}{l}\mathrm{n} \\
\text { Parameters assessed }\end{array}$}} \\
\hline & & \\
\hline Excellent/good result & $100 \%$ & $93 \%$ \\
\hline
\end{tabular}

There were no significant differences in VAS scores at rest, activities of daily living, time of return to work or sports, or satisfaction between the 2 groups. There was a statistically significant difference in VAS scores at work and sports and Mayo 12-pt elbow scores between the 2 groups.

n

Parameters assessed

Poor outcome

Baseline QuickDASH score

Follow-up QuickDASH score

Percentage QuickDASH $<20$
Arthroscopy offers a small but significan improvement in outcome. All cases from 2002 to 2005 were open surgery; after 2005 , all but one were arthroscopic. Follow-up periods were not consistent ( 3 to $6 \mathrm{y}$ ). 
Table 2. Continued

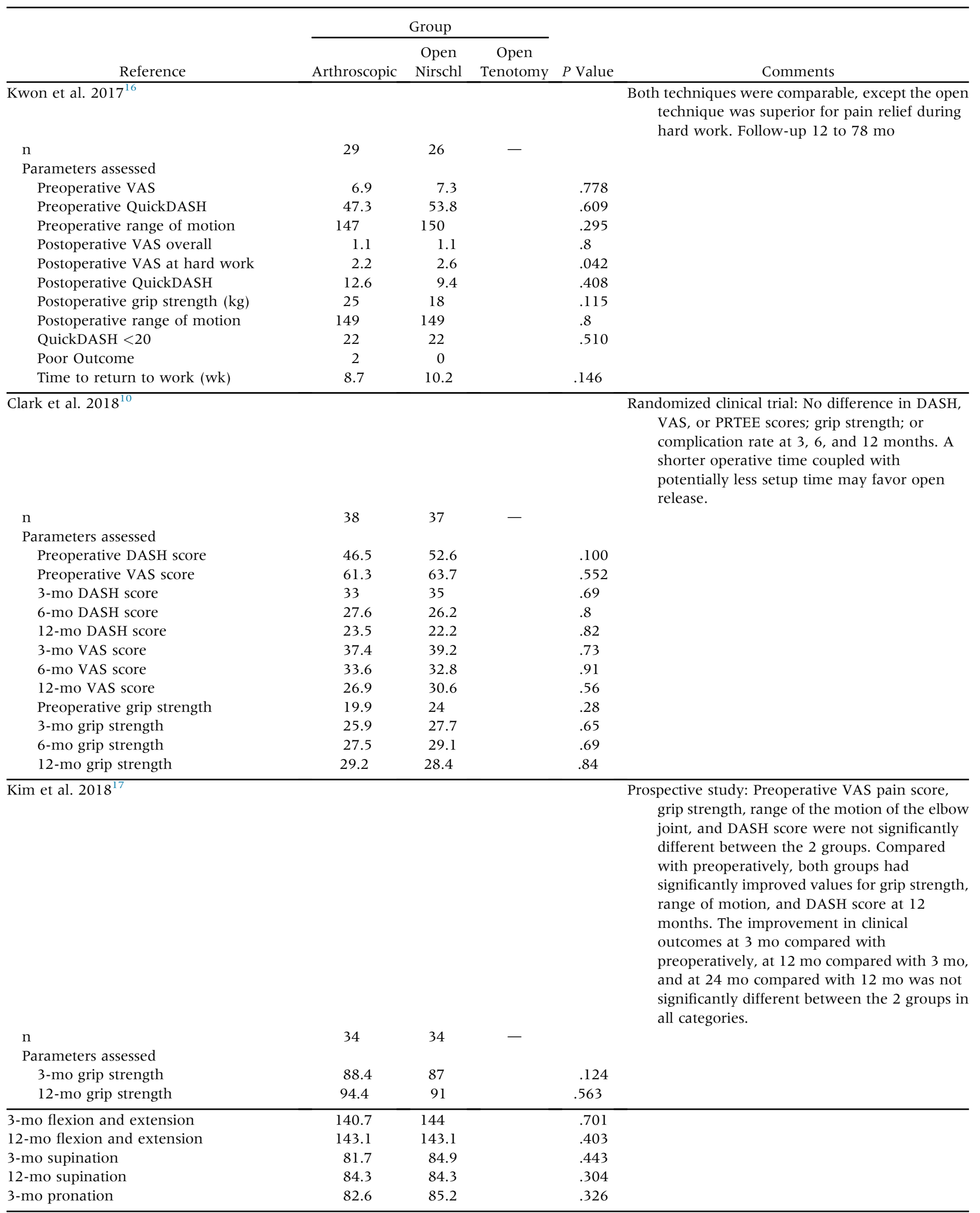

DASH, Disabilities of the Arm, Shoulder and Hand; VAS, visual analog scale. 
The earlier recovery in arthroscopic groups can largely be attributed to the minimally invasive nature of the procedure. Compared with open surgery, where bigger incisions and larger exposure require more time for healing, arthroscopic procedures generally have less downtime. As seen in our study, the mean time taken for complete pain relief was 7.4 weeks in the arthroscopic group and 9.5 weeks in the open group. This shows that the often-used 6-week period may be insufficient to pick up any difference between the 2 surgical groups.

There are very few comparative studies of arthroscopic versus open techniques. ${ }^{9-17}$ In our review, we found 9 studies that compared arthroscopic and open surgical techniques (Table 1). The open techniques in these studies were mainly ECRB tenotomy and Nirschl procedure. In the current study, we performed ECRB reinsertion. To the best of our knowledge, no studies have compared ECRB reinsertion to arthroscopy.

All studies reported improvement after surgery for both open and arthroscopic groups. ${ }^{9-17}$ When comparing the 2 techniques, most authors compared the long-term results, which were overall similar, with minor aberrations., ${ }^{911-16}$ Stapleton and Baker ${ }^{9}$ and Peart et al. ${ }^{11}$ reported earlier return to work with the arthroscopic group; however, statistical analysis was not done. Solheim et al. ${ }^{15}$ reported statistically significant improvement in QuickDASH score in the arthroscopic group, and the percentage of patients with excellent results (QuickDASH $<20$ ) were significantly higher in the arthroscopic group. In contrast, Kwon et al. ${ }^{16}$ reported that the open technique was superior for pain relief during hard work, compared with the arthroscopic group. Kim et al. ${ }^{17}$ reported that open group had significantly greater improvements in grip strength and VAS, whereas the arthroscopic group had significantly greater improvement in pronation. Besides these aberrations, all other parameters were similar between the 2 groups (Table 2).

Most of these studies looked at the long-term outcome of the procedures, usually after many years. ${ }^{13,15,16}$ At the long-term follow up, determining the relative contribution of surgery compared with the natural self-limiting course of the disease is difficult. Hence, the current study attempted to look at the short-term outcomes-TPR, TFD, JAMAR change at 6 weeks, and VAS at 6 weeks-and the incidence of unfavorable outcomes.

In the present study, overall success was good in both groups, with 61 of 72 patients having favorable outcome. Five patients had persistence of pain, and 7 patients developed recurrence within a year. These were calculated together as unfavorable outcomes. Although there were no significant differences in the incidence of unfavorable outcomes between the 2 groups, it is noteworthy that the arthroscopic group had almost exclusively recurrences (4 recurrences), whereas the open group had more persistence of pain rather than recurrence. This difference may be due to an overlap of prolonged postsurgical pain and early recurrence in the open group. The arthroscopic group has shorter postoperative pain; therefore, the recurrence would be noted as a separate event. It is for this reason we combined both recurrent pain and persistent pain together as unfavorable outcomes.

Our incidence of persistent and recurrent pain is similar to those reported in the literature. Stapleton and Baker ${ }^{9}$ reported $20 \%$ failure in both open and arthroscopic groups. Szabo et al. ${ }^{13}$ reported unfavorable outcomes (recurrence or failure) in 7 of 38 open procedures and 3 of 41 arthroscopic procedures. Solheim et al. ${ }^{15}$ reported poor outcomes in $7 \%$ of arthroscopic and $4 \%$ of open surgeries. Kwon et al. ${ }^{16}$ reported poor outcomes in 2 of 29 arthroscopic patients.

The major finding in our study was that the arthroscopic group had significantly better TPR and TFD than the open group. Although Stapleton and Baker ${ }^{9}$ reported that the time taken to return to work was shorter in the arthroscopic group, no statistical data were provided. Peart et al. ${ }^{11}$ reported that the arthroscopic group returned to work at 1.7 months, compared with 2.5 months for the open procedure. Kwon et al. ${ }^{16}$ reported better pain relief with the open technique, and Kim et al. ${ }^{17}$ reported greater grip strength and VAS with the open technique. However, the current study found no significant difference between the groups in terms of JAMAR change at 6 weeks and VAS at 6 weeks.

Another interesting finding of our study was the significant association between worker's compensation and outcome. Many studies have shown worse outcome for patients associated with WC issues. Grewal et al. ${ }^{18}$ reported that after arthroscopic ECRB release, patients with WC took twice as long to return to work. They also noted that 7 of 23 WC patients were not able to return to work by 42 months. ${ }^{18}$ Balk et al., ${ }^{19}$ in their series of open extensor origin release, reported that $24 \%$ of patients with WC changed jobs because of persistent complaints versus $4 \%$ of non-WC patients. Baker et al., ${ }^{20}$ in their series of 42 arthroscopic releases, reported greater levels of pain with work in WC patients.

The findings in our study are similar to these. We found that WC patients took a significantly longer time to get complete pain relief and get back to full duty. The underlying reasons may be related to the repetitive motion involved with the job, time taken to change jobs, or financial gain.

Open debridement and reattachment as well arthroscopic debridement are equally efficacious in terms of JAMAR at 6 weeks, VAS at 6 weeks, and incidence of unfavorable outcome. However, the arthroscopic technique has quicker pain relief and earlier return to work. Unfavorable outcomes had a significant association 
with WC. WC patients also had delayed return to work and longer duration of pain. Among the 3 variables studied (type of surgery, smoking, and WC), open surgery and WC adversely impacted outcome, whereas smoking had no significant impact.

Most patients had satisfactory outcome after either of the surgeries. No significant difference in unfavorable outcome was seen between the 2 techniques, proving that both techniques are equally efficacious in treatment. Providing a control arm (continued conservative management or sham surgery) would have potentially added further clinical relevance; however, this could not be done in our practice.

\section{Limitations}

Our study is not without its limitations. First, this study is limited by its retrospective design, in which important data can be missing. Second, because patients were not randomized, there is the potential for selection bias. Third, the study groups were not matched, which makes it difficult to know what variables were affecting the outcomes. After analysis, we noted that the study groups were uneven, with more smokers in the WC group than in the arthroscopic group, which could have affected our results. Fourth, only 2 techniques among the many available techniques were compared. Fifth, although our sample size was comparable to previous studies, it might still have been underpowered. A larger sample size would have probably been desirable. Finally, the type of work done by the patients was not assessed.

\section{Conclusion}

The arthroscopic technique offers advantages of earlier return to work and shorter recovery period, along with additional advantages of joint inspection and ability to treat coexisting pathologies. Worker's compensation has a significant association with unfavorable outcome. WC patients also had a longer time to return to full duty and time for complete pain relief.

\section{References}

1. Nirschl RP, Pettrone FA. Tennis elbow. The surgical treatment of lateral epicondylitis. J Bone Joint Surg Am 1979;61:832-839.

2. Faro F, Wolf JM. Lateral epicondylitis: Review and current concepts. J Hand Surg Am 2007;32:1271-1279.

3. Laban MM, Iyer R, Tamler MS. Occult periarthrosis of the shoulder: A possible progenitor of tennis elbow. Am J Phys Med Rehabil 2005;84:895-898.

4. Gregory BP, Wysocki RW, Cohen MS. Controversies in surgical management of recalcitrant enthesopathy of the extensor carpi radialis brevis. J Hand Surg Am 2016;41: 856-859.
5. Rose NE, Forman SK, Dellon AL. Denervation of the lateral humeral epicondyle for treatment of chronic lateral epicondylitis. J Hand Surg Am 2013;38:344-349.

6. Wang AW, Erak S. Fractional lengthening of forearm extensors for resistant lateral epicondylitis. ANZ J Surg 2007;77:981-984.

7. Almquist EE, Necking L, Bach AW. Epicondylar resection with anconeus muscle transfer for chronic lateral epicondylitis. J Hand Surg Am 1998;23:723-731.

8. Ahmad Z, Siddiqui N, Malik SS, Abdus-Samee M, Tytherleigh-Strong G, Rushton N. Lateral epicondylitis: A review of pathology and management. Bone Joint $\mathrm{J} \mathrm{Br}$ 2013;95:1158-1164.

9. Stapleton TR, Baker CR Jr. Arthroscopic treatment of lateral epicondylitis: A clinical study. Arthroscopy 1996;12: 365-366.

10. Clark T, McRae S, Leiter J, Zhang Y, Dubberley J, MacDonald P. Arthroscopic versus open lateral release for the treatment of lateral epicondylitis: A prospective randomized controlled trial. Arthroscopy 2018;34:3177-3184.

11. Peart RE, Strickler SS, Schweitzer KM Jr. Lateral epicondylitis: A comparative study of open and arthroscopic lateral release. Am J Orthop (Belle Mead NJ) 2004;33: 565-567.

12. Rubenthaler F, Wiese M, Senge A, Keller L, Wittenberg RH. Long-term follow-up of open and endoscopic Hohmann procedures for lateral epicondylitis. Arthroscopy 2005;21:684-690.

13. Szabo SJ, Savoie FH 3rd, Field LD, Ramsey JR, Hosemann CD. Tendinosis of the extensor carpi radialis brevis: An evaluation of three methods of operative treatment. J Shoulder Elbow Surg 2006;15:721-727.

14. Yan H, Cui GQ, Liu YL, Xiao J, Yang YP, Ao YF. [A randomized comparison of open and arthroscopic Nirschl debridement for refractory lateral epicondylitis]. Zhonghua Wai Ke Za Zhi 2009;47:888-891.

15. Solheim E, Hegna J, Øyen J. Arthroscopic versus open tennis elbow release: 3 - to 6-year results of a case-control series of 305 elbows. Arthroscopy 2013;29:854-859.

16. Kwon BC, Kim JY, Park KT. The Nirschl procedure versus arthroscopic extensor carpi radialis brevis débridement for lateral epicondylitis. J Shoulder Elbow Surg 2017;26: 118-124.

17. Kim DS, Chung HJ, Yi CH, Kim SH. Comparison of the clinical outcomes of open surgery versus arthroscopic surgery for chronic refractory lateral epicondylitis of the elbow. Orthopedics 2018;41:237-247.

18. Grewal R, MacDermid JC, Shah P, King GJ. Functional outcome of arthroscopic extensor carpi radialis brevis tendon release in chronic lateral epicondylitis. J Hand Surg Am 2009;34:849-857.

19. Balk ML, Hagberg WC, Buterbaugh GA, Imbriglia JE. Outcome of surgery for lateral epicondylitis (tennis elbow): Effect of worker's compensation. Am J Orthop (Belle Mead NJ) 2005;34:122-126.

20. Baker CL Jr, Murphy KP, Gottlob CA, Curd DT. Arthroscopic classification and treatment of lateral epicondylitis: Two-year clinical results. J Shoulder Elbow Surg 2000;9: 475-482. 